\title{
Status Dystonicus: A Rare, but a Serious Movement Disorders Emergency
}

Priyantha Herath ${ }^{*}$, Kaitlin Krebs and Souvik Sen

Department of Neurology, School of Medicine, University of South Carolina, Columbia SC 29201, USA

*Corresponding author: Priyantha Herath, Department of Neurology, School of Medicine, University of South Carolina, Columbia SC 29201, USA, Tel: 8035456050; Email: Priyantha.Herath@uscmed.sc.edu

Rec date: Nov 03, 2014, Acc date: Nov 27, 2014, Pub date: Nov 29, 2014

Copyright: () 2014 Herath $\mathrm{P}$, et al. This is an open-access article distributed under the terms of the Creative Commons Attribution License, which permits unrestricted use, distribution, and reproduction in any medium, provided the original author and source are credited.

\begin{abstract}
Status dystonicus is a rare, but truly a potentially life threating movement disorders emergency. Recognizing this condition requires awareness, and also knowledge of phenomenological and clinical characteristics. We describe here a patient who was recently diagnosed with this condition, and the clinical features as well as treatment options.
\end{abstract}

Keywords: Movement disorders emergency; Status dystonicus; Etiology; Management

\section{Introduction}

Status dystonicus is one of the rare movement disorders emergencies [1]. In general, this condition is seen in patients with primary dystonia. However, as in this case, patients with secondary dystonia, including drug induced or tardive dystonias, can also develop rare, life-threatening, unremitting, generalized dystonic spasms [2,3]. This has been termed status dystonicus or dystonic storm.

Usually, status dystonicus occurs after intercurrent infection, alteration in anti-psychotic medications, or, more often, for no obvious reason. A well recognized trigger is the paradoxical worsening of dystonias that can occur during initiation of chelating treatment in Wilson's disease [4]. This condition may be more common in children with dystonia due to various metabolic disorders [5]. Those who develop the condition are at risk of respiratory or airway compromise, aspiration pneumonia, or renal failure from secondary rhabdomyolysis $[1,3,5-7]$. In addition to the usual infectious markers and the metabolic panel, creatine kinase, electrolytes, and renal function should be carefully monitored closely during dystonic storm. Even without any of these complications, there is the potential that the patients can succumb to exhaustion.

\section{Clinical Presentation}

A 46 year-old woman, with a history of Paranoid Schizophrenia, was seen in a consultation at an inpatient mental health facility because of abnormal movements. These symptoms started insidiously 2-3 months ago. Patient reported that her initial symptoms included a sense of tension in the neck muscles, and then, a physical, sustained pulling causing the patient to involuntarily turn her neck towards her left shoulder. After a short while, the pulling sensation would subside. She reported that the pulling sensation would occur with certain actions, but not with others. Over time, the symptoms have spread to other contiguous body parts and eventually affecting her entire body. As such, there are other involuntary, semi-sustained movements in her trunk, limbs, face, and mouth. On occasion, her eyes would involuntarily close for a period of time. Because her neck and trunk have a tendency to pull back, she finds it difficult to walk. Recently she discovered a sensory trick to relieve some of the neck posturing: the act of touching the inside of her mouth, seems to effectively relieve some of these movements (Figure 1).

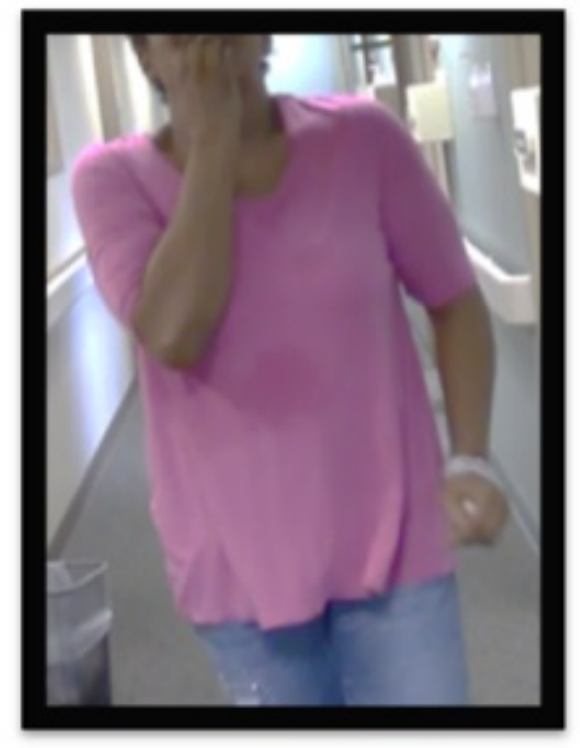

Figure 1: Patient touching the inside her mouth, that effectively relieves the neck from being pulled back. This is a classic sensory sign, or "geste antagoniste".

Because of the history of paranoid schizophrenia, there is protracted history of prior exposure to DA antagonists over many years. However, since the onset of her involuntary movements, she has been entirely weaned off of these agents. She is now an inpatient being treated for severe depression with an Selective Serotonin Reuptake Inhibitors (SSRI). She is being considered for Electro convulsive shock treatment (ECT). A review of her medical history revealed only a strong family history of schizophrenia present in her Mother and Sister. 


\section{Neurological Exam}

General and systems exams, along with her vitals, were found to be normal. She was awake and alert but was in severe physical distress. Neurological examination revealed a certain degree of apathy and a low affect. We did not detect any clear symptoms of thought disorder of hallucinations or delusions. There was no evidence of decreased short-term memory, attention span, or general muscle strength. Patient presented with no limitation of visuo-spatial functions or dysexecutive functions at the time of the examination. We found cranial nerves II-XII to be intact and deep tendon reflexes were $2+$ symmetrically. Her motor strength and coordination were noted to be normal.

\section{Movement Examination}

Patient was found not to be hypomimic and hypophonic. Blink rate was diminished. Her saccades were normal. There was a positive glabellar tap but there were no other frontal release signs. Speech was soft-normal with some dysphonia on occasion. Abnormal movements were presented with multiple ongoing semi- sustained posturing including transient, semi-repetitive movements that were generalized. Blepharospasm and intermittent oromandibular dystonias, including some jaw opening postures, were present. Various degrees of cervical posturing that were mainly Left torticollis with some Left shifting laterocollis and retrocollis. In addition, there were appendicular and truncal dystonias but no camptocormia was noted.

Ongoing tremors were not present. Her muscle tone was normal but could be worsened with activation. These finding were bilateral. Mild bradykinesia was presented with finger taps, as well as with repetitive hand movements, heel taps and toe tapping. Patient experienced difficulty standing from a seated position and required support from her arms to push off the chair. When walking (Figure 2), her base was wide and she had an irregular stance with dystonic intrusions in to her cadence. Mild postural stooping, diminished arm swing bilaterally and slow turns with hesitancy were also present but patient had no gait apraxia or freezing of gait. When waking backwards dystonic lower extremity posturing was much less. A pull test revealed that the patient would have fallen had not been caught by the examiner.
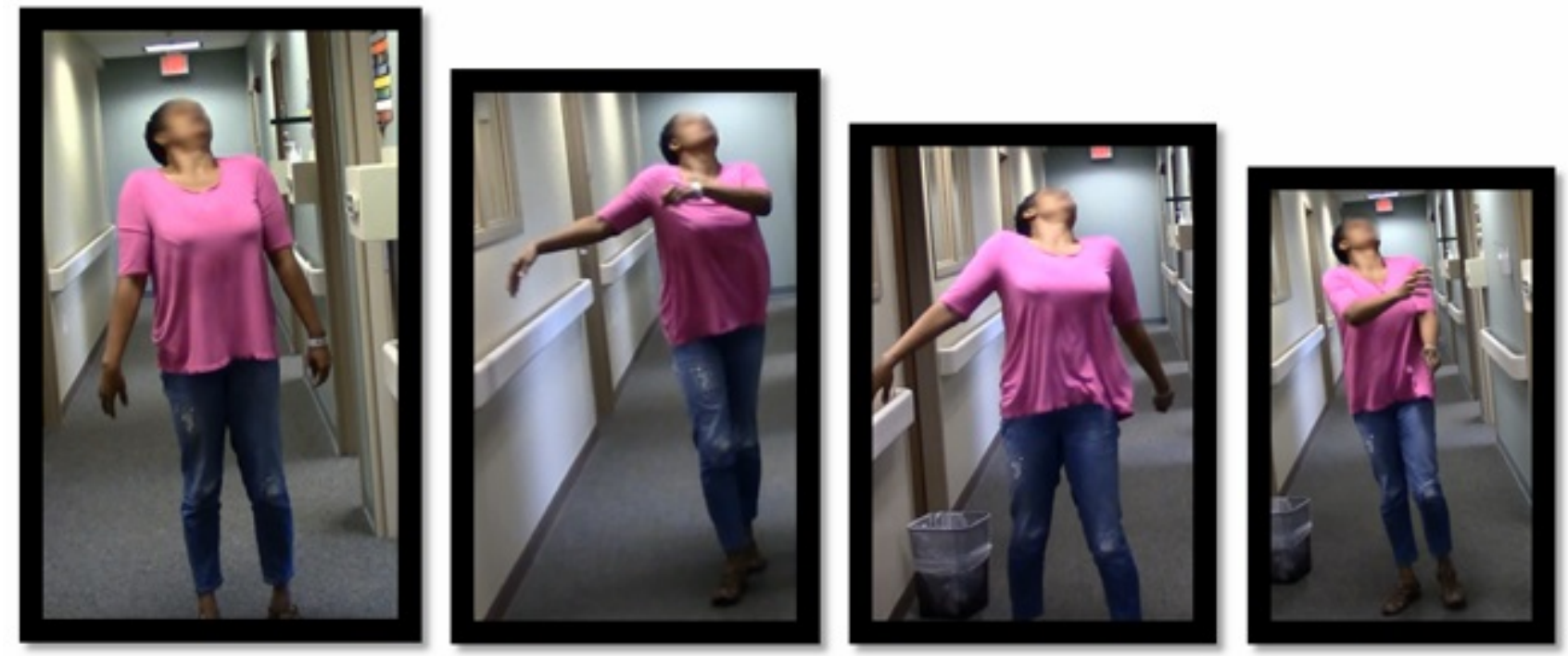

Figure 2: Dysonic posturing of the neck, and limbs in patient with Status Dystonicus.

Based on the clinical history exposure to DA antagonists, along with the insidious onset of repetitive and prolonged sustained involuntary movements that has persisted for at least 3 months now, a diagnosis of Status Dystonicus was made. Other differentials included tardive dystonia, complex tardive tics or tic status and a psychogenic movement disorder.

\section{Management}

The response to conventional drug treatment is often poor [6]. Oral trihexyphenydyl and benzodiazepines, are the main agents commonly used, as was the case for this patient. Other agents such as levodopa, tetrabenazine, baclofen, propranolol are some times used but with varying degrees of symptomatic control. In some instances, combinations of oral tetrabenazine and dopamine blockers could be useful. When the severity of symptoms surpass the usual measures, sedation and paralysis with ventilation in the setting of an ICU is sometimes necessary to prevent the secondary complications $[3,7]$. In rare instances, if medical therapy fails, functional surgery such as bilateral ventrolateral thalamotomy, unilateral pallidotomy, or bilateral pallidal stimulation may be the last potential option for these patients $[8,9]$.

\section{Discussion}

This paper describes a rare but a true movement disorder emergency. Since this is a rare disorder, neurologists have limited expose to the symptoms presented in patients with movement disorders. If not recognized, many secondary complications can occur, or can lead to prolonged suffering of the patient [2]. For this reason alone, this case serves as an illustrative example. 
Citation: Herath P, Krebs K, Sen S (2014) Status Dystonicus: A Rare, but a Serious Movement Disorders Emergency. J Neurol Disord 2: 193.

Page 3 of 3

The diagnosis is entirely clinical, and therefore, an observant neurologist should be able to include this differential diagnosis in a patient who fits the clinical profile we have described above. No imaging or invasive studies are needed.

Once recognized, in addition to providing supportive care, an opinion of a movement disorders specialist should be sought. At times, having a protocol in place with a treatment algorithm may prove to be useful in optimizing outcomes. If there is inadequate experience in the management of these conditions, patients should be transferred to a tertiary care movement disorders center.

\section{Acknowledgement}

This research work has been supported by NIH grant: R01NS062754

\section{References}

1. Mishra D, Singhal S, Juneja M (2010) Status dystonicus a rare complication of dystonia. Indian Pediatr 47: 883-885.

2. Baxter P (2013) Status dystonicus: under-recognized and under-treated. Dev Med Child Neurol 55: 99.
3. Manji H, Howard RS, Miller DH, Hirsch NP, Carr L, et al. (1998) Status dystonicus: the syndrome and its management. Brain $121: 243-252$.

4. Svetel M, Sternic N, Pejovic S, Kostic VS (2001) Penicillamine-induced lethal status dystonicus in a patient with Wilson's disease. Mov Disord 16: 568-569.

5. Jamuar SS, Newton SA, Prabhu SP, Hecht L, Costas KC, et al. (2012) Rhabdomyolysis, acute renal failure, and cardiac arrest secondary to status dystonicus in a child with glutaric aciduria type I. Mol Genet Metab 106: 488-490.

6. Allen NM, Lin JP, Lynch T, King MD (2014) Status dystonicus: a practice guide. Dev Med Child Neurol 56: 105-112.

7. Mariotti P, Fasano A, Contarino MF, Della Marca G, Piastra M, et al. (2007) Management of status dystonicus: our experience and review of the literature. Mov Disord 22: 963-968.

8. Jech R, Bares M, Urgosík D, Cerná O, Klement P, et al. (2009) Deep brain stimulation in acute management of status dystonicus. Mov Disord 24: 2291-2292.

9. Walcott BP, Nahed BV, Kahle KT, Duhaime AC, Sharma N, et al. (2012) Deep brain stimulation for medically refractory life-threatening status dystonicus in children. J Neurosurg Pediatr 9: 99-102. 